\title{
Velocity gradients and relative depth perception
}

\author{
MYRON L. BRAUNSTEIN and GEORGE J. ANDERSEN \\ University of California, Irvine, California 92717
}

\begin{abstract}
The effectiveness of velocity gradients in providing relative depth information was assessed using random dot patterns translating horizontally. The gradients simulated two planes meeting at a horizontal line at the center, and subjects judged whether the center was the nearest or farthest part of the display. Accuracy increased with maximum dot speed, exceeding $90 \%$ in conditions combining the highest speed $\left(10.4^{\circ} / \mathrm{sec}\right)$ and longer of two display durations (10 sec) with unrestricted fixation. Separate experiments examined a rotational component perceived in the motion of the planes and the latency in reporting a rigid organization of the displays. Possible reasons for the chance accuracy found by Farber and McConkie (1979) and alternative explanations of the effect of maximum dot speed on accuracy are discussed. A model is presented that accounts for the effects of dot speed and display duration on the accuracy of relative depth judgments.
\end{abstract}

The velocities with which the projections of objects move across the retina have long been regarded as providing information about the relative distances of objects from the observer (Helmholtz, 1925). Gibson (1950) has proposed that a gradient of velocities is important in surface perception. Gibson, Gibson, Smith, and Flock (1959) compared displays of two velocities with displays of velocity gradients. The texture elements were reported to vary in distance in both cases, but only in the gradient case were the faster elements consistently reported to be closer. That study used a shadow projection technique that produced a texture gradient corresponding to the velocity gradient, leaving unanswered the question of whether relative depth can be perceived on the basis of a velocity gradient alone. Braunstein (1968) used computer-generated displays to separate texture and velocity gradient information. Velocity information clearly dominated texture information in determining judged slant, but the question of whether reversals in relative depth (i.e., the slower elements perceived as closer) could occur with slant information available only from the velocity gradient was not addressed directly.

Two recent studies used displays of velocity gradients carried by texture elements that were randomly and uniformly distributed across the field of view. Rogers and Graham (1979) studied velocity gradients representing slanted and corrugated three-dimensional surfaces. The translation of the dot patterns across a display monitor in one condition was linked to the subject's head movements relative to a stationary monitor and in another condition was linked to move-

This research was supported, in part, by National Science Foundation Grant BNS-76-81499. The authors are grateful to Jerry Keys, who developed the computer programs for generating the stimuli. ment of the monitor relative to the subject's stationary head. Subjects were able to identify the threedimensional shape and to judge the relative depth of portions of the surface with a high degree of accuracy. Rogers and Graham concluded that, under these head movements or monitor movement conditions, motion parallax provided accurate relative depth information.

Farber and McConkie (1979), on the other hand, concluded that motion parallax was not effective in providing relative depth information for a stationary observer, even when a gradient of velocities was displayed. Their displays were random dot patterns translating horizontally. Velocity gradients were produced by either decreasing or increasing the dot velocities from the center of the screen to the top and bottom (see Figure 1). This simulated two slanted planes forming an angle at a horizontal line bisecting the display, with the angle pointed either towards or away from the observer. Subjects were asked to indicate whether the center was nearer or farther away than the top and bottom edges. Farber and McConkie failed to find a consistent relationship between the relative depth implied by the gradient and subjects' judgments and concluded that velocity gradient information was not sufficient for the perception of relative depth (i.e., the sign or direction of depth).

The present study investigated the conditions under which velocity gradients are sufficient for the perception of relative depth. The gradients used were of the same general type studied by Farber and McConkie: Dots distributed randomly across the display moved horizontally, with dot velocity either increasing or decreasing from the center of the display to the top and bottom edges. Two characteristics of these gradients were selected for detailed study on the basis of preliminary observations of a variety of gradients. The first was the translation speed. Flock (1964) had 


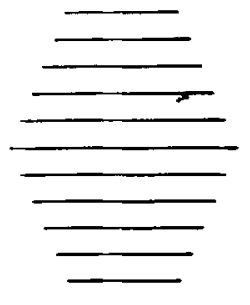

$\mathbf{0}$

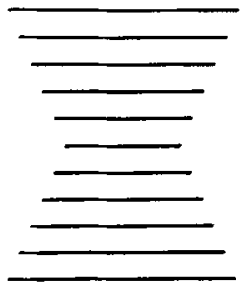

$b$
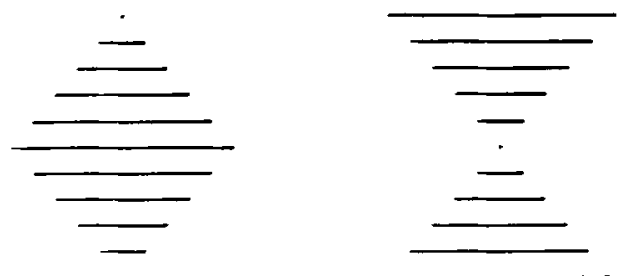

c

Figure 1. The four velocity gradients used in the present research. Gradients $c$ and $d$ were used by Farber and McConkie (1979). Line lengths represent relative velocities.

previously used translation speed as a variable in a study of slant perception for textured planes translating horizontally and had found the same high degree of correspondence of judged slant to physical slant at all of the speed levels studied. His stimuli, however, displayed texture gradients that corresponded to the velocity gradient information, and his results do not show whether variations in translation speed affect depth judgments based on velocity gradients alone. Our preliminary observations suggested a strong relationship between translation speed and the accuracy of relative depth judgments.

In most previous studies of perceived depth from velocity gradients, all texture elements were in motion in the same direction (Braunstein, 1968; Flock, 1964; Gibson et al., 1959). In Farber and McConkie's study, the slowest elements were stationary. Stationary elements in a velocity gradient representing a slanted plane would correspond to infinitely distant elements. Unlike gradients in which all elements move in the same direction, gradients that include stationary elements do not display a common motion component. The second characteristic of velocity gradients investigated in the present study was the presence or absence of stationary texture elements, leading to the absence or presence of a common motion component.

The first experiment examined the relationship of maximum translation speed and the accuracy of relative depth judgments. (For the displays used in this paper, "relative depth" judgments are defined as center-near vs. center-far discriminations, "correct" judgments are defined as judgments of the faster moving dots as nearer, and accuracy is defined as the proportion of correct judgments.) Translation speeds were selected on the basis of pilot studies to encompass a range of accuracies. Each maximum translation speed was paired with a minimum speed that was half the maximum speed or zero, resulting in the presence or absence of common motion. The first experiment was also intended to clearly demonstrate the existence of stimulus conditions under which highly accurate relative depth judgments could be made solely on the basis of velocity gradient information.

\section{EXPERIMENT 1}

\section{Method}

Subjects. The subjects were 23 students in lower division psychology courses at the University of California, Irvine, who received extra credit for their participation. Vision of at least $20 / 40$ (Snellen eye chart) was required in the eye used in the experiment. Data from one subject was not used because of failure to follow instructions.

Design. There were four independent variables: (1) the velocity of the fastest dots, or "maximum speed" $(2.6,5.2$, or $10.4 \mathrm{deg} /$ sec); (2) the velocity of the slowest dots, or "minimum speed" (half the maximum speed or zero); (3) the gradient direction, increasing from top and bottom to center ("center near") or decreasing from top and bottom to center ("center far"); and (4) the translation direction (left or right). Each subject received two replications of each of the 24 combinations of the four variables.

Stimuli. The stimuli were 4810 -sec (240-frame) computergenerated 16-mm motion-picture sequences. The sequences were generated one frame at a time on a Digital Equipment VR-14 display scope, recorded on Kodak Plus- $X$ film using an Automax camera, and printed on high-contrast film. Each sequence showed 326 white dots translating horizontally against a black background. The 48 sequences were arranged randomly with a 5-sec (120-frame) ISI.

The dot velocities were computed as if the dots were projections of points on two slanted planes meeting at a horizontal line bisecting the field of view. The angle formed by the intersecting planes was either the closest or the most distant portion of the display, according to whether a "center near" or a "center far" condition was being generated. For half of the displays in each condition, an angle of intersection was selected for the two planes that produced a 2:1 ratio between the fastest and slowest dots. This also yields a $2: 1$ ratio between the simulated observer's distance to the closest points in the display and the observer's distance to the farthest points. The angle that produces this ratio is $33.7 \mathrm{deg}$ for the center-near condition (Figure 2a) and $18.4 \mathrm{deg}$ for the center-far condition (Figure $2 b$ ). (Center-far displays that matched the center-near displays in angle rather than in ratio of maximum-to-minimum velocities were included in a pilot study, but there was no indication that these displays would provide useful additional information.) For the remaining half of the displays in each condition, the maximum translation speeds were set equal to those in the first half, but the minimum speed was set to zero. This corresponds to an angle between the planes equal to the angle of view in the center-near condition (Figure $2 \mathrm{c}$ ) and to parallel horizontal planes ("meeting" at the horizon) in the center-far condition (Figure 2d). Figure 3 shows examples of center-near and center-far displays.

Apparatus. A 16-mm projector (L-W Athena) was used to present the film. Two of three shutter apertures were covered to prevent the perception of multiple images (Braunstein, 1966). The film was projected through a glass window onto a translucent screen (Polacoat) in a separate room. The dot and background luminances at the screen were .4 and $.007 \mathrm{~cd} / \mathrm{m}^{2}, A .4$ neutral density filter was located in the viewing tube to enhance the perception of the background as totally dark.

The subject viewed the screen monocularly from the opposite side at a distance of $1.8 \mathrm{~m}$ through a tube that restricted the field 


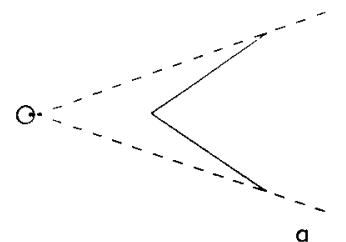

a
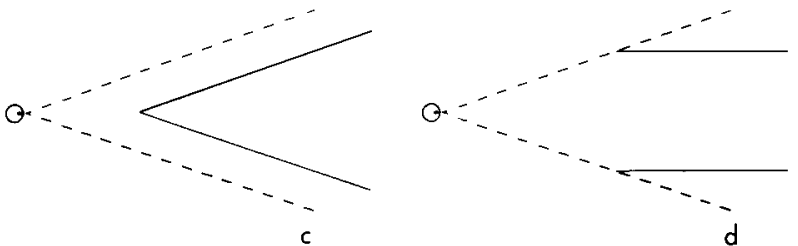

Figure 2. Side views of three-dimensional configurations corresponding to the four projected velocity gradients in Figure 1.
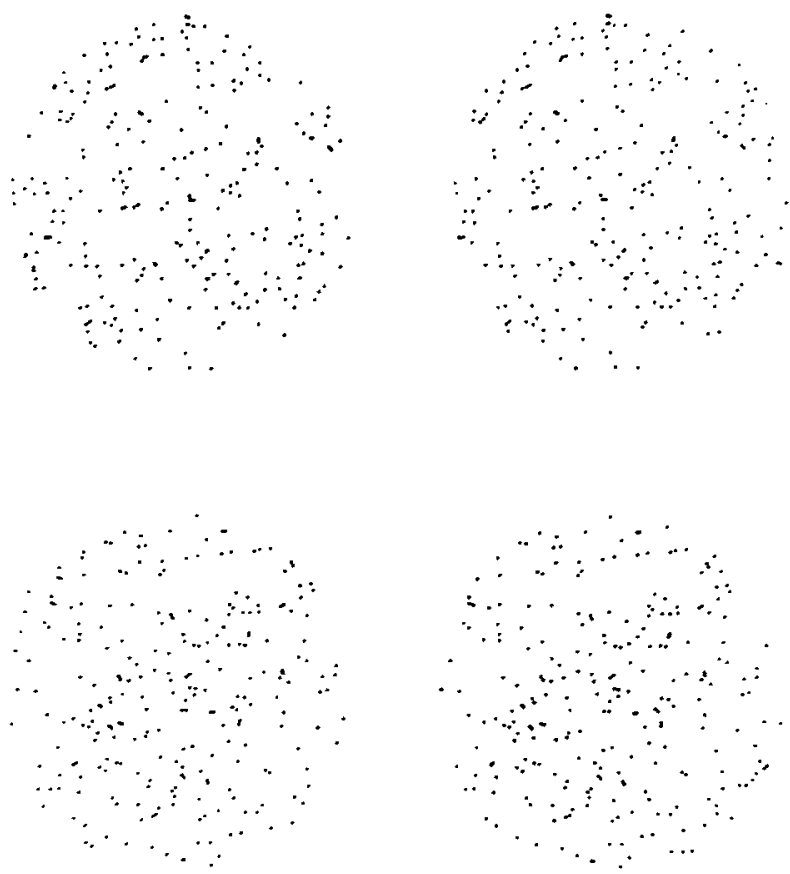

Figure 3. Dot patterns of the type presented on successive frames of motion-picture sequences displaying center-near (top pair) and center-far (bottom pair) gradients. The gradients may also be seen by viewing the two frames in each pair stereoscopically.

of view to a circle $1.2 \mathrm{~m} \mathrm{(37} \mathrm{deg)} \mathrm{in} \mathrm{diameter.} \mathrm{Approximately} 256$ dots were visible through the tube, each dot subtending a visual angle of $.5 \mathrm{deg}$.

A $37 \times 23 \mathrm{~cm}$ piece of black posterboard, bent along its axis to an angle of approximately $80 \mathrm{deg}$, served as a demonstration model. Small white dots were unsystematically positioned on both sides of the posterboard.

Procedure. The subjects were run individually. Each subject was instructed to indicate, immediately after each display disappeared, whether the center dots appeared nearer or more distant by saying "center near" or "center far." These two conditions were demon- strated using the posterboard model. The subject's room was then made totally dark and the stimulus film was started. A different order of presentation was obtained for each of the 22 subjects by running the film in either the forward or reverse direction, with 11 different (equally spaced) starting positions used in each direction. A total of 53 sequences were presented to each subject. The last five sequences were the same as the first five, which were treated as practice trials. At the end of the session, the subject was asked a series of questions about the displays, beginning with "what did you think you were looking at?"

\section{Results and Discussion}

A total of 1,056 judgments were collected, 48 from each of 22 subjects. The overall proportion of correct judgments was .84. The proportion of center-far judgments was .56. The proportions of correct responses for maximum speeds of $2.6,5.2$, and $10.4 \mathrm{deg} /$ sec were $.75, .88$, and .94 with a minimum dot speed one-half the maximum speed and $.73, .84$, and .91 with a minimum speed of $0 \mathrm{deg} / \mathrm{sec}$. The effects of the four independent variables (maximum dot speed, minimum dot speed, gradient direction, and direction of translation) were examined in an analysis of variance, using the number of correct judgments by each subject for each stimulus condition $(0,1$, or 2$)$ as the dependent measure. The frequency of correct responses increased significantly $[F(2,42)=20.3$, $\mathrm{p}<.01$ ] with maximum dot speed. The main effect of minimum dot speed was not significant $[F(1,21)=$ $3.03, p>.05]$. As might be expected from the higher overall proportion of center-far responses, there were significantly more correct judgments for center-far stimuli $(.90)$ than for center-near $(.78)[F(1,21)=$ $17.2, p<.01]$. The main effect of direction of translation was not significant $[\mathrm{F}(1,21)<1]$.

There was a significant interaction between maximum dot speed and gradient direction $[F(2,42)=$ $5.33, \mathrm{p}<.01]$. The effect of gradient direction was smallest at the fastest speed, with the proportion of correct responses exceeding .90 for both center-near and center-far gradients (see Figure 4). There were four other significant interactions: Gradient Direction by Direction of Translation $[F(1,21)=4.98, p<.01]$, Minimum Speed by Direction of Translation $[F(1,21)$ $=7.79, \mathrm{p}<.05]$, Maximum Speed by Minimum Speed by Direction of Translation $[F(2,42)=5.71$, $\mathrm{p}<.01]$, and the four-way interaction $[\mathrm{F}(2,42)=$ $6.94, \mathrm{p}<.01]$.

Responses to the debriefing questions indicated that the displays were highly realistic. When asked what they thought they were looking at through the viewing tube, 13 of the 22 subjects described threedimensional objects, 3 described two-dimensional projections (a computer terminal, a film, slides), and 6 expressed uncertainty or gave ambiguous responses.

The high levels of accuracy found in this experiment leave little doubt that velocity gradient information in isolation (or, more precisely, in conflict with texture, relative size, and relative brightness in- 


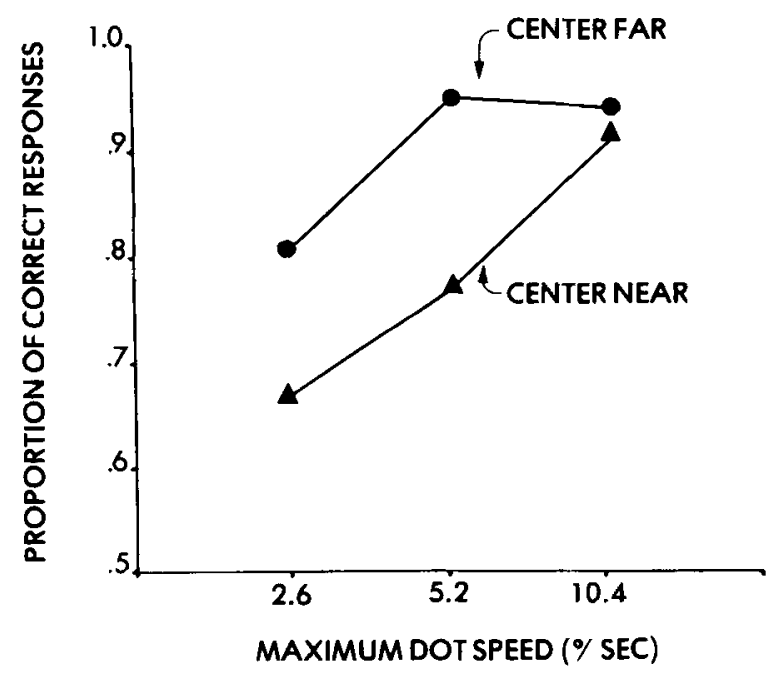

Figure 4. Interaction of maximum dot speed and gradient direction in determining accuracy of relative depth judgments (Experiment 1).

formation that implied equidistance of the dots) is sufficient to elicit correct judgments of direction of slant. The major new finding of this experiment was the importance of the maximum translation speed in determining the effectiveness of the gradient information. Possible reasons for this effect will be examined in the next four experiments. The finding of greater accuracy for center-far responses is not surprising, as these displays are analogous to real-world scenes in which the ground plane and sky meet at a horizon near the vertical center of the visual field. There is no similarly commonplace analogy to real-world scenes for the center-near displays.

\section{EXPERIMENT 2}

The effect of maximum dot speed on the accuracy of relative depth judgments might reflect one of several underlying relationships. On the one hand, either displayed or projected speed may have a direct effect on accuracy. On the other hand, the effective variable might be a function of speed and display duration. Examples of variables jointly determined by speed and duration include the maximum horizontal translation of a dot, the maximum number of dots passing a given point in the display, and the total number of times the fastest dots enter and leave the field of view. If one or more of these variables determined accuracy, a reduction in display duration would reduce the accuracy found in Experiment 1. Of course, any reduction in observation time for a complex stimulus might reduce the accuracy of the required judgments, but, if the critical variable is a product of speed and duration, accuracy should remain a function of this product. This possibility was tested in Experiment 2, which was a replication of Experiment 1, with the display duration reduced from 10 to $5 \mathrm{sec}$. The 5 -sec duration was also selected for comparability to Experiment 2 of Farber and McConkie (1979). The accuracy found in Experiment 1 of the present series was considerably higher than that found in their experiment. Experiment 2 will consider the possibility that the longer display duration used in Experiment 1 contributed to the higher accuracy of the relative depth judgments.

\section{Method}

Subjects. The subjects were 13 students from the same source as in Experiment 1. The vision criterion employed was the same as that in Experiment 1. Data from one subject were not used in the analysis because of failure to follow instructions.

Stimuli. The displays were the same as those used in Experiment 1 , but the duration was reduced to $5 \mathrm{sec}$ and the ISI increased to $10 \mathrm{sec}$.

Apparatus. The apparatus was the same as in Experiment 1, except for the addition of a tachistoscopic shutter and timer to control the stimulus duration.

Procedure. The procedure was essentially the same as in Experiment 1 , except that only six equally spaced starting positions were required in both the forward and reverse film directions to provide different orders of presentation for the 12 subjects.

\section{Results and Discussion}

The results were similar to those of Experiment 1, except for a general reduction in the proportion of correct judgments. The overall proportion of correct judgments was .76. The proportion of center-near judgments was .61. The proportions of correct responses for maximum speeds of 2.6, 5.2, and $10.4 \mathrm{deg} /$ sec were $.73, .74$, and .83 with a minimum dot speed one-half the maximum speed and $.69, .75$, and .81 with a minimum speed of $0 \mathrm{deg} / \mathrm{sec}$. Each of these six proportions is lower than the corresponding proportion in Experiment 1. The proportion of correct judgments was affected significantly by the maximum dot speed $[F(2,22)=5.23, p<.05]$. The main effect of gradient direction was significant $[F(1,11)$ $=8.9, \mathrm{p}<.05$ ], with proportions of correct judgments of .87 and .65 for the center-far and centernear stimuli. The main effects of minimum speed $[F(1,11)<1]$ and of direction of translation $[F(1,11)$ $=1.5, \mathrm{p}>.05]$ were not significant. The only significant interaction was minimum speed with direction of translation $[F(1,11)=9.2, p<.05]$.

The results of Experiments 1 and 2 provide an opportunity to consider the relationship of the maximum extent of the translation to the proportion of correct judgments. The maximum speed of $5.2 \mathrm{deg} /$ sec with a 10-sec display duration (Experiment 1) may be compared with the 10.4-deg/sec speed with a 5-sec duration (Experiment 2). The proportions of correct judgments for these two conditions were .86 and .82. Similarly, the $2.6 \mathrm{deg} / \mathrm{sec}$ condition in Experiment 1 can be compared with the $5.4 \mathrm{deg} / \mathrm{sec}$ condition in Experiment 2. Proportions of .74 were ob- 
tained for both these conditions. Although these two comparisons may not be sufficient as evidence of a relationship between maximum extent of translation and proportion of correct judgments, they do suggest that this hypothesis may merit further consideration.

\section{EXPERIMENT 3}

Although the reduced display duration used in Experiment 2 resulted in reduced accuracy of relative depth judgments in all velocity conditions, accuracy still exceeded the chance levels found by Farber and McConkie (1979). One difference between Experiment 2 and Farber and McConkie's study was the use of fixation instructions in the earlier study. Farber and McConkie noted that eye movements add a uniform flow component to the velocities projected on the retina, resulting in retinal velocities that are not proportional to object distance. This may be taken to imply that the velocity gradients should be registered more accurately with fixation on a stationary point than with free observation. This implication, however, is based on concept of direct perception of retinal motion, which a recent study by Gogel (1980) has effectively contradicted. Other researchers (Festinger, Sedgwick, \& Holtzman, 1976) have suggested that eye movements might be helpful in judgments of relative motion. Experiment 3 introduced a fixation target and fixation instructions similar to those used by Farber and McConkie in order to determine whether fixation could, at least in part, account for their findings of chance accuracy. If the addition of a uniform flow component to the retinal velocities is detrimental to the accurate perception of relative depth from a velocity gradient, accuracy in this experiment should exceed that found in Experiment 1 . If eye movements, on the other hand, are helpful in the registration of a velocity gradient, a decrease in accuracy relative to that obtained in Experiment 1 should be found.

\section{Method}

Subjects. The subjects were 16 students from the same source as in Experiment 1. The vision criterion was the same as that employed in Experiment 1. Data from four subjects who failed to meet a minimum criterion for reported fixation (see Procedure) were not used in the analysis.

Stimuli. The stimulus displays were the same as those in Experiment 1 . In addition, a fixation target consisting of a circle, $1 \mathrm{deg}$ in diameter and with a point at the center, was continuously visible at the center of the projection screen. The target luminance at the screen was $1 \mathrm{~cd} / \mathrm{m}^{2}$.

Apparatus. In addition to the apparatus used in Experiment 1, a Kodak slide projector was used to project the fixation target.

Procedure. The procedure was the same as in Experiment 1, except for the addition of fixation instructions. Subjects were asked to carefully fixate on the point in the center of the circle and not to follow the motion of the dots. Following the experimental session, the subjects were asked to state the percentage of time they did not fixate on the target while the moving dots were present. Data from subjects who reported percentages of $15 \%$ or greater were not used in the analysis. The order of presentation of the displays was the same as in Experiment 2.

\section{Results and Discussion}

As in Experiment 2, the pattern of results for this experiment was similar to that in Experiment 1 except for a general reduction in the proportion of correct judgments. The overall proportion of correct responses was .78 . The overall proportion of centerfar responses was .56. The proportions of correct responses for maximum speeds of $2.6,5.2$, and $10.4 \mathrm{deg} / \mathrm{sec}$ were $.73, .81$, and .83 with a minimum dot speed one-half the maximum speed and $.69, .77$, and .82 with a minimum speed of $0 \mathrm{deg} / \mathrm{sec}$. Each of these six proportions is lower than the corresponding proportion in Experiment 1. The effect of maximum speed was again significant $[F(2,22)=3.50, p<.05]$. The effect of minimum speed was not significant $[F(1,11)<1]$. The effect of gradient direction was significant $[F(1,11)=6.5, p<.05]$, with proportions of .84 for the center-far displays and .71 for the centernear displays. The main effect of direction of translation was not significant $[F(1,11)=4.39, p>.05]$. There were no significant interactions.

The detrimental effect of fixation instructions on the use of velocity gradients in judgments of relative depth suggests that eye movements may play a role in the registration of a velocity gradient. This possibility will be considered in the general discussion.

\section{EXPERIMENT 4}

Subjects in the first three experiments reported (during debriefing) that the displays sometimes appeared to rotate about a vertical axis as well as translate horizontally. The perception of a rotary component in the motion of a translating surface might be expected when the perceived relative distances do not correspond to the distances simulated by the velocity gradients. This misperception of relative distance may be divided into two cases. In the first, there is a reversal of perceived depth. The slowest dots are perceived as closer and the faster dots as more distant. With this reversal of depth, the velocity gradient is not consistent with a horizontal translation. It does, however, roughly approximate a configuration in which the faster dots are rotating about a vertical axis through the slower (or stationary) dots. As the displayed gradient is not completely consistent with a rotary motion, some lack of rigidity must either be tolerated by the visual system or perceived by the observer.

In the second case, rotation is perceived without depth reversal, but the slant of the surfaces is underestimated. The slant of a rigidly translating surface is uniquely determined by the ratio of the fastest to the 
slowest projected velocities, taken over a given visual angle. This relationship is independent of the size and absolute distance of the surface. (Functions relating slant to perspective and visual angle are discussed by Braunstein \& Payne, 1969.) This case may be divided into two subcases. First, when the slowest dots are moving, an underestimation of surface slant should result in a failure of the translatory motion to account for the difference between the fastest and slowest projected velocities across a given visual angle. The addition of a perceived rotational component, again with a vertical axis through the slower dots, could account for an additional portion of the velocity difference.

The other subcase consists of displays with stationary dots. If these dots are not perceived as being infinite in distance, their failure to move is not consistent with a rigid translation. A perceived rotary motion, with a vertical axis through the stationary dots, is an alternative perception. (This perception is not completely consistent with the display, since there are stationary dots spread along a horizontal line, but it seems to occur nevertheless, with the axis of rotation near the center of the display.)

Experiment 4 considered the relationship of perceived rotation to the accuracy of relative depth judgments. Subjects were asked to categorize each display as showing "no rotation," "some rotation," or "mostly rotation." If perceived rotation is associated with perceived depth reversal, "mostly rotation" responses should be associated with incorrect depth judgments, and the frequency of these responses should be determined by the same variable that determines accuracy-maximum dot speed.

\section{Method \\ Subjects. The subjects were 14 students from the same source as in Experiment 1. The vision criterion was the same as that em- ployed in Experiment 1. Data from two subjects were not used in the analysis because of failure to follow instructions. \\ Stimuli and Apparatus. The stimulus and apparatus were the same as in Experiment 1. \\ Procedure. The following procedures were added to the proce- dures used in Experiment 1: Subjects were informed that some of the displays might appear to rotate and that they were to indicate the degree of rotation seen in each display by responding "no rotation," "some rotation," or "mostly rotation." The rotation judgment was made immediately after the relative depth (center- near or center-far) judgment for each display. Examples of ro- tation with center-near and center-far displays were demonstrated using the posterboard model. Order of presentation for the dis- plays was the same as in Experiment 2.}

\section{Results and Discussion}

Rotation judgments. The overall proportions of low-, medium-, and high-rotation judgments were .25 , .47 , and .28 . Since the stimulus conditions producing a maximum impression of rotation were of primary interest, the frequency of high-rotation responses was selected as the dependent variable for an analysis- of variance. The independent variables were the same as in the analyses of the relative depth judgments in Experiments 1-3. Rotation judgments were affected significantly by both maximum speed $[F(2,22)=$ $21.5, \mathrm{p}<.01]$ and minimum speed $[\mathrm{F}(1,11)=19.7$, $\mathrm{p}<.01]$. The mean proportions of high-rotation responses were $.083, .22$, and .55 for maximum speeds of $2.6,5.2$, and $10.4 \mathrm{deg} / \mathrm{sec}$; they were .18 for minimum speeds that were half the maximum speed and .39 for minimum speeds of $0 \mathrm{deg} / \mathrm{sec}$. These results may be contrasted with the results with relative depth judgments as the dependent variable, which did not show a significant effect of minimum speed in any of the experiments. Inspection of the proportions of highrotation responses for the six combinations of maximum and minimum speed (Table 1) shows that these proportions are ordered by the difference between the maximum and minimum speeds in a display. The six velocity combinations yield four differences: 1.3 , $2.6,5.2$, and $10.4 \mathrm{deg} / \mathrm{sec}$. Each of the two intermediate levels is based on two speed combinations. For example, a difference of 5.2 can be produced by a maximum speed of 10.4 and a minimum speed of 5.2 or a maximum speed of 5.2 and a minimum speed of 0 . Table 1 shows that similar proportions of highrotation responses were found with equal speed differences, even when these were based on different combinations of maximum and minimum speeds. The main effects of gradient direction $[F(1,11)=$ 1.30] and of direction of translation $[F(1,11)<1]$ were not significant $(p>.05)$. There were no significant interactions.

Relative depth judgments. The results for relative depth judgments were similar to those in Experiment 1, indicating that the procedure of eliciting a rotation judgment after each depth judgment did not alter the effects of the independent variables on the depth judgments. The overall proportion of correct responses was .82. The proportion of center-far responses was .54. The proportions of correct responses for maximum speeds of $2.6,5.2$, and $10.4 \mathrm{deg} / \mathrm{sec}$ were $.78, .86$, and .93 with a minimum dot speed onehalf the maximum speed and $.70, .78$, and .90 with a minimum speed of $0 \mathrm{deg} / \mathrm{sec}$. Each of these six pro-

Table 1

Relationship of Rotation Judgments to Translation Speed (in Degrees per Second)

\begin{tabular}{|c|c|c|c|}
\hline \multicolumn{3}{|c|}{ Speed } & \multirow{2}{*}{$\begin{array}{c}\text { Proportion of } \\
\text { High Rotation } \\
\text { Judgments }\end{array}$} \\
\hline Maximum & Minimum & Difference & \\
\hline 2.6 & 1.3 & 1.3 & .04 \\
\hline 2.6 & .0 & 2.6 & .12 \\
\hline 5.2 & 2.6 & 2.6 & .09 \\
\hline 5.2 & .0 & 5.2 & .34 \\
\hline 10.4 & 5.2 & 5.2 & .40 \\
\hline 10.4 & .0 & 10.4 & .70 \\
\hline
\end{tabular}


portions exceeds both the corresponding proportion in Experiment 2 and the corresponding proportion in Experiment 3. The main effect of maximum speed was significant $[F(2,22)=12.7, p<.01]$, as was the main effect of gradient direction (center-near vs. center-far) $[F(1,11)=8.5, p<.05]$. The main effects of minimum speed $[F(1,11)=2.25, p>.05]$ and of direction of translation $[F(1,11)<1]$ were not significant. There were no significant interactions.

Comparison of rotation and relative depth judgments. There was some tendency for higher levels of rotation to be reported with incorrect depth judgments. The proportions of low-, medium-, and high-rotation responses for the 101 incorrect depth judgments were $.13, .53$, and .33 . These proportions differed significantly from the proportions of $.27, .45$, and .27 found for the 476 correct responses $\left[\chi^{2}(2)=9.3, p<.01\right]$. Most (.85) of the high-rotation responses made with incorrect depth judgments occurred when center-near displays were reported as center-far.

The sizable proportion of high-rotation responses made with correct depth judgments (.27) indicates that underestimation of surface slant was not an infrequent occurrence for these displays. Underestimation of slant is typical in laboratory experiments with two-dimensional projections and is probably due to "flatness cues" in the displays. In this study, several types of information indicating flatness were deliberately left in the stimuli in order to isolate velocity gradients as the only carrier of depth information. The texture density was uniform in the projected displays, and the dots were equal in size and brightness.

The perception of a rotary component in the motion of a rigid translating surface is not peculiar to twodimensional projections, although it may be exaggerated in such displays. A person looking out of the side window of an automobile traveling through open country may notice apparent rotation of surfaces stretching out into the distance. This is probably due to an underestimation of the greater distances in the scene.

\section{EXPERIMENT 5}

The first four experiments found a consistent effect of maximum dot speed on the accuracy of relative depth judgments. Informal observations during these experiments suggested that there was some latency in the perceptual organization of the displays, analogous to the latency found in the perception of a threedimensional structure in a random dot stereogram. Successive frames of the stimulus film can, in fact, be viewed as random dot stereograms, with the disparity a function of the maximum dot speed in the display from which the frames are extracted. (See Figure 3.) Experiment 5 considers the possibility that maximum dot speed determines the latency with which the display organizes into two rigid planes, and that this latency is related to the accuracy of relative depth judgments. Subjects were asked to indicate when each display appeared to organize into a surface, as well as to provide a center-near or center-far judgment.

\section{Method}

Subjects. The subjects were 15 students from the same source as in Experiment 1. The vision criterion was the same as that employed in Experiment 1. Data from three subjects who did not meet a minimum response criterion (see Procedure) were not used in the analysis.

Stimuli. The stimuli were the same as those used in Experiment 1.

Apparatus. In addition to the apparatus used in Experiment 1, a digital stopclock registered the time elapsed from the opening of a tachistoscopic shutter at the start of each display until the subject pushed a button mounted in a table under the viewing tube.

Procedure. The following procedures were added to the procedures used in Experiment 1: The subjects were informed that at first the displays might appear as random dots moving independently of each other and that after some time the dots might appear to organize into a surface similar to that of the demonstration model. The subjects were asked to press a button as soon as the moving dots appeared to organize into a surface and to then report a center-near or center-far judgment. The subjects were further informed that if a display disappeared before the moving dots appeared to organize into a surface, they should give their relative depth judgments at that time. Data from subjects who failed to indicate that a display had organized into a surface on $25 \%$ or more of the trials were not used in the analysis. The orders of presentation of the displays were the same as in Experiment 2.

\section{Results and Discussion}

Latency. The mean latency in reporting that the displays had organized in depth, for all subjects and stimuli (576 trials), was $3.2 \mathrm{sec}$. This included 28 trials on which no responses were made, recorded as $10 \mathrm{sec}$. The main effects of maximum speed $[\mathrm{F}(2,22)$ $=8.06, \mathrm{p}<.01]$ and of minimum speed $[\mathrm{F}(1,11)$ $=7.72, \mathrm{p}<.05]$ and the interaction of these variables $[F(2,22)=5.06, p<.05]$ were significant. Latency generally decreased with increasing maximum translation speed and was lower when the minimum speed was zero than when it was half the maximum speed. The interaction of maximum and minimum speeds in determining latency is shown in Figure 5. The main effects of gradient direction $[F(1,11)=4.29]$ and of direction of translation $[F(1,11)<1]$ were not significant $(p>.05)$. There were no other significant interactions.

Relative depth judgments. The overall proportion of correct judgments was .78. The proportion of centerfar responses was .55 . The proportions of correct responses for maximum speeds of 2.6, 5.2, and $10.4 \mathrm{deg} /$ sec were $.72, .73$, and .89 with a minimum dot speed one-half the maximum speed and $.66, .81$, and .85 with a minimum speed of $0 \mathrm{deg} / \mathrm{sec}$. When compared with the corresponding proportions in Experiments 1 


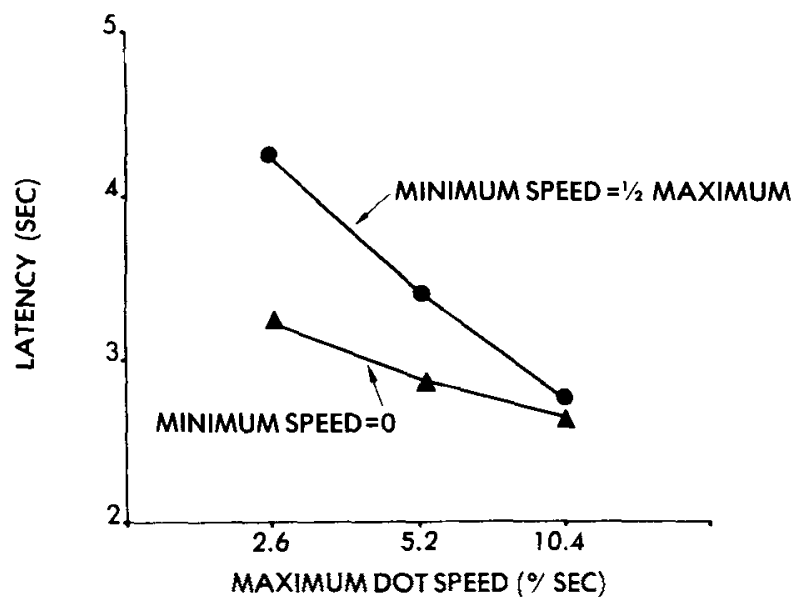

Figure 5. Interaction of maximum and minimum dot speeds in determining latency of display organization (Experiment 5).

and 4, which used the same viewing conditions and display duration, these proportions are lower in 11 of the 12 comparisons. This is not surprising, since the relative depth judgments in this experiment immediately followed the display organization responses, which had a mean latency of $3.2 \mathrm{sec}$. In Experiments 1 and 4, the depth responses were made at the end of the 10-sec display period. The frequency of correct judgments was significantly affected by maximum speed $[F(2,22)=9.25, p<.01]$. The main effects of minimum speed $[F(1,11)<1]$, gradient direction $[F(1,11)=4.04)$, and direction of translation $[F(1,11)$ $<1]$ were not significant $(p>.05)$. There were no significant interactions.

Comparison of latency and relative depth judgments. The mean latency was greater for incorrect depth judgments $(3.95 \mathrm{sec})$ than for correct depth judgments $(3.08 \mathrm{sec})[\mathrm{t}(11)=2.21, \mathrm{p}<.05]$. It should not be concluded from this result, however, that the accuracy of the relative depth judgments was simply a function of how quickly the displays organized. Accuracy in all five experiments was affected by maximum speed but not by minimum speed, while latency was affected by both maximum and minimum speeds, with a significant interaction. Like the highrotation judgments in Experiment 4, but unlike accuracy, latencies are ordered by the differences between maximum and minimum speeds in the displays. This suggests that latency may be related to the tendency of a display to organize with a rotational component, whether or not relative depth is perceived in accordance with the velocity gradient. This hypothesis would have to be tested with the simultaneous collection of relative depth judgments, rotation judgments, and latencies under conditions that would produce a sufficient number of reversals to permit detailed comparisons of correct and incorrect responses.

\section{DISCUSSION}

The first experiment provided two principal conclusions, which were replicated in each of the four additional experiments: First, relative depth can be judged with a high degree of accuracy on the basis of a velocity gradient alone. The overall proportion of correct responses exceeded .75 in each experiment and proportions exceeding .90 were found under some conditions. These high levels of accuracy did not require head movements, texture gradients, or other depth information. Texture density, relative size, and relative brightness in fact provided contradictory information by suggesting that the display was in the frontal plane.

The present results differ from those of Farber and McConkie (1979), who found that subjects were unable to make accurate relative depth judgments with a maximum speed of $2.15 \mathrm{deg} / \mathrm{sec}$ and a minimum speed of $0 \mathrm{deg} / \mathrm{sec}$. This difference in results was probably due to a combination of methodological differences. The display duration was $5 \mathrm{sec}$ in the earlier study, while it was $10 \mathrm{sec}$ in each of the present experiments except Experiment 2. The shorter duration did not reduce accuracy to chance levels in Experiment 2 , but it may have interacted with one or more of the remaining methodological differences in Farber and McConkie's experiment. The possibility of such an interaction is suggested by the finding, in Experiment 5, of a latency exceeding $3 \mathrm{sec}$ in organizing the slower displays as rigid surfaces. With display organization consuming most of the observation period for 5-sec displays, accuracy could be sharply reduced by any procedural difference that either increased the time required to organize a display or reduced the rate at which relative depth information was extracted after the display organized.

There are two procedural differences that could have affected the time required to organize the displays as slanted surfaces in depth. First, Farber and McConkie's subjects had previously responded to another type of display, and this prior experience may have introduced competing display organizations. In these other displays, half of the dots were moving to the left at one velocity and half were moving to the right at another velocity. These displays were perceived as two frontally oriented planes at different depths, with perceived depth unrelated to relative velocity. The subjects in the present experiments had not previously served in any related experiments, and no subject served in more than one experiment.

The second difference was in the shape of the dot field. Both studies used a circular viewing aperture, but in Farber and McConkie's experiment the dots were confined to a rectangular area within the circular area. In the present experiments, the dots filled the circular viewing area. A rectangular border can serve as a cue 
to the flatness of a display, and is likely to reduce the realism of a simulated three-dimensional display.

The rate at which information about the displayed gradient was acquired may have been reduced by the use of a fixation point and fixation instructions. This would be expected if eye movements are important in acquiring this information. The fixation procedure did not result in chance accuracy in Experiment 3 with a 10-sec display duration, but it could have interacted with the shorter duration in Farber and McConkie's experiment. Which combinations of variables are important in reducing the accuracy of relative depth judgments based on velocity gradients would be an appropriate subject of future research.

Other investigators have failed to find a consistent relationship between displayed velocity and judged distance for displays of two (Gibson et al., 1959) or three (Kottas, 1978) velocities. It appears that a gradient of velocities, and probably the perception of a rigid surface, is required for accurate relative depth judgments.

The second major finding in the present study was that the maximum speed of translating display elements is the principal variable determining accuracy of relative depth judgments that are based on a velocity gradient. Maximum speed significantly affected these judgments in each experiment, but minimum speed, on the other hand, never significantly affected accuracy or interacted significantly with maximum speed. This is a surprising finding, since the effectiveness of a velocity gradient might be expected to be a function of the ratio of maximum-to-minimum speeds, of the difference in speeds, or of some other measure of the steepness of the gradient. Any of these possibilities would have produced an effect of minimum speed or an interaction of minimum speed with maximum speed. (An effect of speed difference on accuracy would be expected, however, if the difference were reduced to just detectable levels. The relationship of speed difference to latency of display organization found in Experiment 5 suggests that such displays might fail to organize as slanted surfaces.)

There are a number of alternative explanations of the effect of maximum speed on the accuracy of relative depth judgments. Experiments 2 through 5 provide suggestions as to which explanations may be the more promising, but a definitive answer will require considerably more research. The first explanation would be difficult to test because it is little more than a restatement of the effect: The maximum speed provides a reference point for registering the gradient, and the higher the speed, the more salient the reference point. A more specific version of this explanation is that the maximum speed determines the accuracy with which the direction of translation is perceived. Incorrect registration of direction of translation could result in reversal of perceived depth (Farber \& McConkie, 1979). Informal observations of the stimuli used in the present series of experiments suggest that there was little ambiguity as to the direction of translation, but it may be interesting to collect direction of translation judgments at slower translation speeds.

A possible mechanism by which maximum dot speed might enhance gradient registration is by increasing the number of saccadic eye movements that occur during the observation of a display. If the subject tracked the motion of the faster dots across the display, there would be primarily pursuit eye movements in the direction of the translation, with occasional saccades in the opposite direction. These saccades should provide information about eye movements, such as retinal smear (Festinger \& Holtzman, 1978 ), in addition to information directly associated with the pursuit movements. Saccades would be necessary when a dot that was being tracked reached the edge of the display and disappeared and a new dot or cluster of dots farther back along the motion path was selected for tracking. Since the display diameter was $36 \mathrm{deg}$, the fastest dots would not fully traverse the widest portion of the display in the $2.6-\mathrm{deg} / \mathrm{sec}$ condition but would do so almost three times in the 10.4-deg/sec condition.

The maximum translation speed may affect the maintenance of alternative perceptual organizations. A specific organization associated with incorrect relative depth judgments was that the slower dots were closer to the observer and the faster dots were rotating about the slower ones (thus accounting for their greater projected speeds). This alternative organization is contradicted by the linear, rather than sinusoidal, projected velocity of the dots over their entire horizontal path and the failure of the projected velocity to decrease at the ends of the path. There are more opportunities to observe this discrepancy at the higher speeds, since dots will enter and leave the display more frequently. The incorrect alternative perceptual organization should be maintained more frequently with a shorter display period and with attention directed toward the center of the display, since both these conditions would reduce the opportunity to register discrepant information in the linear paths of the translating dots. The results of Experiments 2 and 3 are consistent with this explanation. Accuracy was reduced with shorter display durations, and was consistent with the total extent of translation, or the total frequency with which dots would enter or leave the display. Accuracy also was reduced with fixation instructions. This explanation suggests that relative depth judgments for displays in which high rotation is reported will be more accurate at higher maximum dot speeds. Considering the 163 high-rotation responses in Experiment 4, the distribution of the 130 correct responses among the three maximum velocity conditions was .07, .22, and .72. For the 33 incorrect 
responses, the distribution was $.21, .42$, and .36 . This deviates significantly from the proportions found for the correct responses $\left[\chi^{2}(2)=19.8, p<.01\right]$. Although the frequency of incorrect responses is too small for a definitive conclusion, the expected drop in incorrect high-rotation responses does appear to take place at the highest maximum dot speed.

A third explanation of the effect of maximum dot speed is that maximum speed determines ease of perceptual organization of the moving dots into a rigid surface. This possibility is contradicted by the results of Experiment 5. Latency in reporting display organization corresponds to the difference between the maximum and minimum speeds rather than to the maximum speed alone. Mean latencies across the six speed combinations studied have the same rank order as high-rotation judgments. Displays with large speed differences may have organized quickly with a rotation component whether or not this organization provided an accurate perception of relative depth.

The faster displays may have produced a blur pattern that was useful in registering the gradient (Harrington, Harrington, Wilkins, \& Koh, 1980). The speeds studied, however, are not usually associated with blur. In addition, the use of a three-bladed projector shutter with two apertures covered yielded an on/off ratio of 1:5. This low on/off ratio should have helped to minimize blur. A blur-pattern explanation would not account for the effectiveness of maximum speed rather than the range or ratio of speeds. However, as previously discussed, the number of eye movements opposite to the direction of translation should have been a function of the maximum speed and display duration. These eye movements would increase the velocity of the dots across the retina, possibly creating a blur pattern that would be effective in judging relative depth. On the other hand, saccadic suppression (Matin, 1974) could have eliminated this information, and the presence of a significant effect of maximum speed with a fixation point in Experiment 3 indicates that this effect cannot be attributed entirely to eye movements. Research is needed on the type of eye movements that occur during observation of velocity gradients to clarify their relationship to blur patterns.

A final possibility is that the accuracy of relative depth judgments is actually determined by the difference between the maximum and minimum speeds, but a decrement in accuracy occurs when the minimum speed is zero. This decrement could obscure the effect of speed difference by lowering accuracy for speed differences based on lower maximum speeds with minimum speeds of zero. For example, the maximum/minimum pairs of $(5.2,0)$ and $(10.4,5.2)$ have equal speed differences, but the latter resulted in higher accuracy. Rather than the higher accuracy's being due to the higher maximum speed of the second pair, it may be that the accuracy of judgments for the first pair was reduced by the zero minimum speed. The possibility of a decrement in performance with a minimum speed of zero is suggested by the especially large conflict that this velocity gradient produces with texture density, size, and brightness information. A zero velocity implies an effectively infinite distance, and should be associated with texture elements that are densely packed and barely visible as individual elements. Since this does not occur in these displays, alternative perceptions (e.g., rotation of the other dots around the stationary ones) may have a higher probability of occurrence. Another possible reason for a decrement in performance with a minimum speed of zero is the lack of a common motion component in these displays (Johansson, 1976). This may have increased the difficulty of perceiving an overall translatory motion. The possibility that the speed difference may be the important variable would have to be tested with a set of speed differences produced by a number of combinations of maximum and minimum speeds, including zero and nonzero minimum speeds, and various ratios of the maximum speeds to the nonzero low speeds.

The following model is proposed on the basis of the results of the present series of experiments: (1) Velocity gradients that display small speed differences may fail to organize as rigid surfaces, resulting in some chance judgments of near-far relationships. (2) Displays with larger speed difference may produce a reversed organization with a large rotational component. (3) As the maximum displayed velocity increases, the reversed rotational organization will become harder to maintain. Thus, the probability that a display organizes should be a function of display duration and speed difference, the probability that an organized display appears to rotate should be a function of speed difference, and the probability that a display that appears to rotate is not perceived in reverse when the subject responds should be a function of maximum dot speed and observation time prior to the response.

A detailed version of the model, presented in Table 2 , accounts for the effect of maximum speed on accuracy and for the drop in accuracy with shorter display durations. Other models could undoubtedly account for these results as well. The purpose of presenting this model is to suggest the types of parameter variations and other conditions that require further study. The model suggests, for example, that the time course of perceived organization may be informative. The continuous recording of judgments of relative depth, rotation, and rigidity, possibly along with eye movements, over longer observation periods should be useful in more precisely defining the processes underlying the effectiveness of velocity gradients in depth perception. 
Table 2

An Information Processing Model of Depth Judgments From Translating Velocity Gradients

I. Is a rigid organization perceived?

(Probability computed as a function of log speed difference)

A. If yes, is there a rotation component?

(Probability computed as a function of speed difference)

1. If yes, is near-far reversed?

(Probability computed as a function of log maximum speed)

a. If yes, is it time to respond?

i. If yes, respond incorrectly.

ii. If no, return to I.A.1.

b. If no, respond correctly.

2. If no, respond correctly.

B. If no, is it time to respond?

1. If yes, respond correctly or incorrectly on a probabilistic basis.

2. If no, return to $I$.

\section{REFERENCES}

Braunstein, M. L. Interaction of flicker and apparent movement. Journal of the Optical Society of America, 1966, 56, 835-836.

Braunstein, M. L. Motion and texture as sources of slant information. Journal of Experimental Psychology, 1968, 78, 247-253.

Braunste in, M. L., \& Payne, J. W. Perspective and form ratio as determinants of relative slant judgments. Journal of Experimental Psychology, 1969, 81, 584-590.

FARber, J. M., \& McConkiE, A. B. Optical motions as information for unsigned depth. Journal of Experimental Psychology: Human Perception and Performance, 1979, 5, 494-500.

Festinger, L., \& Holtzman, J. D. Retinal image smear as a source of information about magnitude of eye movement. Journal of Experimental Psychology: Human Perception and Performance, 1978, 4, 573-585.

Festinger, L., Sedgwick, H. A., \& Holtzman, J, D. Visual perception during smooth pursuit eye movements. Vision Research, 1976, 16, 1377-1386.

FLock, H. R. Some conditions sufficient for accurate monocular perceptions of moving surface slants. Journal of Experimental Psychology, 1964, 67, 560-572.

Gibson, E. J., Gibson, J. J., Smith, O. W., \& Flock, H. Motion parallax as a determinant of perceived depth. Journal of Experimental Psychology, 1959, 58, 40-51.
GiBson, J. J. The perception of the visual world. Boston: Houghton Mifflin, 1950.

Gogel, W. C. The sensing of retinal motion. Perception \& Psychophysics, 1980, 28, 155-163.

Harrington, T. L., Harrington, M. K., Wilkins, C. A., \& Кон, Y. O. Visual orientation by motion-produced blur patterns: Detection of divergence. Perception \& Psychophysics, 1980, 28, 293-305.

Helmholtz, H. Physiological optics (Vol. 3). Rochester, N.Y: Optical Society of America, 1925.

JohAnsson, G. Spatial-temporal differentiation and integration in visual motion perception. Psychological Research, 1976, 38, 379-393.

KotTas, B. L. The visual three-dimensional information from motion parallax (Doctoral dissertation, Vanderbilt University, 1978). Dissertation Abstracts International, 1978, 39, 3022-B. (University Microfilms No. 7823458)

MAtin, E. Saccadic suppression: A review and an analysis. Psychological Bulletin, 1974, 81, 899-917.

Rogers, B., \& Graham, M. Motion parallax as an independent cue for depth perception. Perception, 1979, 8, 125-134.

(Received for publication August 19, 1980; revision accepted January 9,1981 .) 